\title{
$\begin{array}{ll}\text { Research Square } & \begin{array}{l}\text { Preprints are preliminary reports that have not undergone peer review. } \\ \text { They should not be considered conclusive, used to inform clinical practice, } \\ \text { or referenced by the media as validated information. }\end{array}\end{array}$
}

\section{Quantitative Evaluation of Upright Posture by X-Ray and 3D Stereophotogrammetry With an Original Marker Placement Protocol in Late Onset Pompe Disease.}

Paolo De Blasiis ( $\sim$ paolodeblasii@gmail.com )

University of Trieste Department of Medical Surgical and Health Sciences: Universita degli Studi di Trieste Dipartimento Universitario Clinico di Scienze Mediche e Chirurgiche e della Salute https://orcid.org/0000-0002-1317-7994

Allegra Fullin

University of Campania Luigi Vanvitelli: Universita degli Studi della Campania Luigi Vanvitelli

Mario Sansone

University of Naples Federico II: Universita degli Studi di Napoli Federico II

Luca Del Viscovo

University of Campania Luigi Vanvitelli: Universita degli Studi della Campania Luigi Vanvitelli

Filomena Napolitano

University of Campania Luigi Vanvitelli: Universita degli Studi della Campania Luigi Vanvitelli

Chiara Terracciano

University of Campania Luigi Vanvitelli: Universita degli Studi della Campania Luigi Vanvitelli

Giacomo Lus

University of Campania Luigi Vanvitelli: Universita degli Studi della Campania Luigi Vanvitelli

Mariarosa Anna Beatrice Melone

University of Campania Luigi Vanvitelli: Universita degli Studi della Campania Luigi Vanvitelli

Simone Sampaolo

University of Campania Luigi Vanvitelli: Universita degli Studi della Campania Luigi Vanvitelli

\section{Research}

Keywords: Late Onset Pompe Disease, 3D stereophotogrammetry, X-Ray, upright posture, standing sagittal alignment, marker placement protocol

Posted Date: January 14th, 2021

DOI: https://doi.org/10.21203/rs.3.rs-144072/v1

License: @ (1) This work is licensed under a Creative Commons Attribution 4.0 International License. Read Full License 


\section{Abstract}

Background: Late Onset Pompe Disease (LOPD) is an autosomal recessive muscular disorder characterized by prevailing weakness of trunk and pelvic girdle muscles that causes ventilatory insufficiency and postural abnormalities. The most common myopathy phenotype described clinically in LOPD is the Limb Girdle and Diaphragmatic Pattern; spinal deformities, as hyperlordosis, hyperkyphosis and scoliosis diagnosed by $x$-Ray exam, have been reported in about a third of LOPD patients. The non-specific clinic onset, the similarity of the LOPD phenotypes with other myopathies, inter-individual heterogeneity and the lack of any disease hallmark, make early diagnosis challenging and, if enzyme replacement therapy does not begin timely, about $60 \%$ of patients develops severe motor and ventilatory disabilities.

Aim of our study was to quantitatively assess the upright posture in a sibship of LOPD adults by x-Ray(xR) and 3D Stereophotogrammetry (St), considered the gold standard to measure spinal angles and whole-body posture respectively, in order to better identify specific alterations more likely to be present in a homogeneous group.

Results: Statistical analysis of St parameters showed a larger ankle, knee, elbow, dorsal, S2-C7, heel-S2-C7, heel-S2-nasion angles and a lower sagittal vertical axis (SVA) than healthy controls.

Moreover, $\mathrm{xR}$ analysis highlighted a lower occipito-cervical, C2-C7 cervical and Cobb dorsal angles, and a trend to lower lumbar lordosis and SVA compared to normal values.

Pearson's coefficient analysis was carried on in order to evaluate the correlation between $x \mathrm{R}$ and St sagittal spino-pelvic parameters and significant correlation was found in dorsal and lumbar angles calculated using XR markers placed on spiny apophysis, XR centre of vertebral bodies, Cobb method and St markers.

Conclusions: This is the first study that quantitatively assesses standing whole-body alignment and postural abnormalities in LOPD. These postural alterations are not easily detectable during clinical examination and might be useful to early identify LOPD patients and to facilitate differential diagnosis with other proximal myopathies. Moreover, our St-mks placement protocol showed high reliability to assess any sagittal angles and, being non-invasive as compared to $\mathrm{xR}$, is advisable to investigate and monitor the course of the disease and the response to treatment.

\section{Background}

Pompe Disease is a rare autosomal recessive neuromuscular disorder, due to mutations of the GAA gene codifying the lysosomal acid alpha14 -glucosidase enzyme (acid maltase, $G A A$ ), and characterized by glycogen accumulation primarily in skeletal, cardiac and smooth muscle [1]. Mutations causing reduction of musculoskeletal GAA activity below $1 \%$ the normal, lead to very severe infantile form (IOPD) which is incompatible with life if not treated early with enzyme replacement therapy (ERT) [2]. Levels of GAA activity between $1 \%-25 \%$ the normal, cause late onset form (LOPD) which may occur at any age from 2 to 70 years. This mild form has a better prognosis for life expectance than IOPD form, but about $35 \%$ of patients is confined to wheelchair and/or requires mechanical ventilation before the age of 65 , and all LOPD patients show postural and motor disabilities [3]. Recent clinical trials demonstrated that ERT leads to better functional results if it is established earlier and associated with rehabilitation [4,5]. Unfortunately, LOPD diagnosis occurs after an average delay of 10 years, owing to the variability of clinical manifestations, the rarity of the condition and the overlap of signs and symptoms with other neuromuscular diseases [6].

The phenotypic spectrum of LOPD is quite broad considering the different age of onset, (varying from early infancy to late adulthood), the heterogeneity of musculoskeletal involvement and the rate of disease progression, even in patients carrying the same mutations of GAA. Despite these differences, a recent systematic review reported that about $80 \%$ of LOPD patients showed a clinical triad that consists of a primary involvement of the pelvic girdle, respiratory and trunk musculature [7]. LOPD patients were also classified into three clinical subgroups, the Limb Girdle and Diaphragmatic Weakness (LGDW), the Rigid Spine Syndrome, Scoliosis and Low Body Weight (RSS) and the Cardiocerebrovascular patterns, in relation to their shared prevailing symptoms [8].

MRI studies revealed an involvement of para-spinal lumbar muscles (multifidus, longissimus, iliocostalis muscles) in pre-symptomatic patients with iperCKemia as the only manifestation, whereas abdominal rectus, psoas, iliacus and posterior muscle of the thigh were further involved in symptomatic patients [9]; the severity of muscle changes at MRI-T1w score strongly correlated with muscle weakness, exercise intolerance, decreased endurance, fatigue, difficulties during transitions between positions, reaching upright standing (Gowers' maneuver), ambulation, running and climbing stairs $[7,9]$.

The loss of muscular function is associated to skeleton alterations as osteoporosis and spinal deformities. A third of LOPD patients showed scoliosis and only a quarter of them had an adult-onset form; moreover, more than half of LOPD patients with scoliosis had been diagnosed by 
$\mathrm{xR}$ and, in these subgroup, kyphosis and lordosis abnormalities were respectively found in $13.9 \%$ and $15.9 \%$ of patients, even if the type of abnormalities was never specified [10].

To the best of our knowledge, only one study assessed the stabilometric parameters in LOPD using a force platform, but no study has ever analysed quantitatively whole-body standing alignment in LOPD patients [11].

Based on these observations, aim of our study was to quantitatively assess postural abnormalities in a sibship of LOPD adults by gold standard methods: $x$-Ray for spinopelvic sagittal angles and 3D Stereo-photogrammetry for whole-body upright posture using an original markers placement protocol. This instrumental evaluation could identify specific postural parameters useful for early and differential diagnosis with other proximal myopathies, in order to start ERT as soon as possible and to quantitatively monitor the treatment outcomes.

\section{Methods}

\section{Subject populations}

Seven LOPD siblings, sharing the same GAA mutation compound, were recruited in Neuromuscular Disease Centre of the University of Campania "L.Vanvitelli" and assessed using clinical scales (Medical Research Council and Range Of Movement), X-Ray (xR) and 3D Stereophotogrammetry (St) in upright posture. Fourteen healthy individuals, age and sex-matched, were used as controls for St-parameters.

\section{Data gathering}

Quantitative evaluation of upright postural alignment was performed using the SMART stereo-photogrammetric system (BTS BioengineeringMilano, Italy); a new markers placement protocol (titled DB Total, Figure 1, A) was specifically designed for better studying the spine, the head, the arms on the sagittal plane and the feet on the frontal plane, extending standard Helen Heyes M.M. Protocol [12] (Figure 1, A -blue markers). Markers were always placed by the same expert operator on nasion (Ns), frontozygomatic suture (FZs), spiny apophysis of C7 -T7 -T12 -L3 -L5 -S2, acromioclavicular joint (ACj), epicondylus humeri (eH), ulnar styloid (Us), anterior-superior iliac spine (ASIS), greater trochanter (gT), medial (mEF) and lateral (IEF) epicondylitis femoris, fibular head (Fh), medial (mM) and lateral (IM) malleolus, $I^{\circ}-I^{\circ}$ and $\mathrm{V}^{\circ}$ metatarsal head (MtH), heel $(\mathrm{He})$ bilaterally.

Moreover, radiographic spinopelvic sagittal parameters [16-23] were assessed by x-Ray (OperaG80HF - GMM - Seriate (BG) - Italy), positioning patient in perfect orthostatic position and acquiring plain film radiograms in a lateral view; radiopaque markers were placed on the spiny apophysis of C7-T7-T12-L3-L5-S2 (Figure 2, A) in according to DB-Total protocol, to study the correlation between St and xR parameters.

\section{Data pre-processing and analysis of standing data}

Data were exported from BTS and processed in Matlab (MATLAB Release 2017b, The MathWorks, Inc., Natick, Massachusetts, United States) with ad hoc developed software.

We analysed the whole-body sagittal angles concerning Helen Hayes M.M. protocol (as showed in Figure1 A, C) and other parameters concerning DB Total (as showed in Figure $1 \mathrm{~B}, \mathrm{C}$ ).

Using Matlab, radiological angles were evaluated and plotted on the x-Ray images, as shown and explained in figures 2 B, C. To this aim, an expert radiologist manually identified landmark points (vertebral corners, the profile of the sacral plate, and the profiles of femoral heads) on radiographic images using Osirix; landmarks coordinates were input into ad hoc developed Matlab script.

\section{Statistical analysis}

As the aim of the study was to individuate the upright posture parameters more suitable to a characterisation of the LOPD patients compared to the healthy population, for each St and xR parameter the distribution between the two populations was analysed. For clinical and St parameter healthy population was recruited as control group, while for xR normative values were found in literature [16,17,18]. In particular, baseline features and ROM values have been tested for clinical differences between patients and controls using Wilcoxon-Mann-Whitney test and t-test, respectively.

Moreover, Wilcoxon-Mann-Whitney test was used to evaluate separation between patients and healthy subjects for St-parameters, while t-test was used for $\mathrm{xR}$ parameters. P-values less than 0.05 were considered statistically significant different.

Pearson's correlations coefficients (including confidence intervals) have been computed between St and xR parameters in order to assess the reliability of DB Total markers placement protocol (high correlation coefficient $>0.7$ and $<-0.7$ ). All statistical analysis has been performed in $\mathrm{R}$ [13]. 


\section{Results}

Baseline features of LOPD patients (L) and controls (C) are: gender= 4F, 3M in L; $8 \mathrm{M}, 6 \mathrm{~F}$ in C; mean age $=57.1 \pm 6.5$ in L; $46.7 \pm 14.9$ in C; mean $\mathrm{BMI}=26.2 \pm 4.2$ in $\mathrm{L} ; 23.4 \pm 3.0$ in $\mathrm{C}$; no significant difference was found between two groups.

LOPD group showed a lower strength in flex-extensor trunk and tibialis anterior muscles (average MRC=2), while the other dorsiflexors ankle (extensor digitalis and hallucis longus) were not compromised; moreover, a significant lower active e passive dorsiflexion (a-and p-ROM) of ankle was found (Table 1, Figure 3).

As regard the evaluation of upright posture, stereophotogrammetric analysis showed significant differences in sagittal parameters, with a larger dorsiflexion ankle, flexion knee, dorsal, S2-C7, Heel-S2-C7 and Heel-S2-Nasion angles and a lower Sagittal Vertical Axis (SVA) in the Pompe group compared to the healthy controls (Table 2).

About radiographic spinopelvic measurements, significant differences were found in sagittal angles with a reduction of occipito-cervical (OC2), C2-C7 cervical (C2-C7-CA) and Cobb Dorsal angles (C-DA) and a trend of reduction of SVA in LOPD patients (Table 3) respect to the normal values.

Moreover, a strong correlation was found between $x R$ and St parameters: 1) dorsal and lumbar angles calculated using xR markers placed on spiny apophysis (xR-mks) and St markers (St-mks); dorsal angles calculated using 2) St markers (St-mks) and xR centre of vertebral bodies (xR-crb), 3) xR-mks and xR-crb and 4) xR-mks and Cobb Angle (Table 4).

\section{Discussion}

The aim of the study was to quantitatively assess the muscular weakness of specific anatomic districts and related postural alterations in upright standing of LOPD patients. Our results showed a deficit of trunk muscles, in accordance to previously reported [1,7,9], but also of tibialis anterior although extensor digitalis and hallucis longus were not compromised (Table 1). This condition masks the deficiency of tibialis anterior, never mentioned in literature, and explains the characteristic morphology of the patients' feet during active and passive ankle dorsiflexion that result decreased (Figure 3, Table 1).

Moreover, this is the first study that evaluate whole-body standing alignment by 3D-stereophotogrammetry and, at the same time, spinopelvic sagittal angles using $x$-Ray, in LOPD patients.

An original marker placement protocol (DB Total protocol, Figure 1, A), created ad hoc extending standard Helen Hayes M.M. protocol (Figure 1, A-blue markers) in order to study new specific stereophotogrammetric parameters (Figure $1 \mathrm{~B}, \mathrm{C}$ ), was introduced to better analyse the postural abnormalities of LOPD patients. In particular, LOPD group showed a significant larger dorsal angle, indicating a flattening of the dorsal curve, and a trend of lumbar hypolordosis, in disagreement to the literature that previously reported an excessive kyphosis and/or lordosis [7,8,10]. Moreover, a larger S2-C7 angle and a lower Sagittal Vertical Axis revealed a C7 position posterior to the sacrum; these findings, associated to a larger dorsiflexion ankle, flexion knee, Heel-S2-C7 and Heel-S2-Nasion angles (Table 2), showed a typical sagittal upright posture of LOPD patient, as a result of a biomechanical compensation strategy of the spinal extensor muscles deficit $[9,14,15]$.

Radiographic spino-pelvic results showed a significant lower Cobb Dorsal Angle (Table3) of Pompe group respect to normative values $[16,17,18]$ confirming the reduction of dorsal kyphosis emerged in stereophotogrammetric analysis; moreover, a significant lower 0-C2 and larger C2-C7 cervical angles were found in LOPD patients, underlining a straightening of the cervical lordosis.

No significative difference was found for Cobb Lumbar angle, but only a trend of hypo-lordosis in accordance with St-results. Finally, only a reduction trend of SVA was found compared to normal values.

Other studies [24] analysed sagittal spine parameters introducing a larger number of markers placed on the spine than standard protocols, but no correlation study was performed between stereophotogrammetric and radiological sagittal angles. Instead, in our study a strong correlation was found (Table 4), highlighting a high reliability of DBTotal protocol in matching markers to spinous processes of the reference vertebrae and in detection of dorsal angles; a lower correlation was found in lumbar angles probably due to the higher thickness of the adipose tissue present in this area.

The results of the present study should be evaluated and considered with respect to some limitations. The relatively small sample size of the populations recruited, due to the low incidence of the rare disease, prevents to any definitive conclusion because of small statistical power. Investigation over larger population and/or meta-analysis are required. Nevertheless, the genetic homogeneity of the LOPD patients, the accuracy of our markers placement protocol demonstrated by $x$-Ray exam and the exclusive choice of sagittal stereophotogrammetric parameters [25,26], make our results reliable. Further studies, considering these quantitative outcome measures, would be needed to better 
evaluate not only static but also dynamic postural alterations during motor tasks that stress LOPD-specific weak muscles, in order to compare them with other myopathic phenotypes.

\section{Conclusions}

Our study is the first to evaluate quantitatively the standing sagittal alignment of LOPD patients and to highlight postural characteristics difficult to identify on clinical examination.

The knowledge of these specific postural alterations might be important for the early and differential diagnosis of LOPD with other proximal myopathies.

Moreover, our St-markers placement protocol showed high reliability to assess dorsal angles and whole-body sagittal alignment (St-HSN and St-HSC) and might be a useful non-invasive procedure to investigate and monitor the course of the disease and the response to treatment.

\section{Declarations}

Ethics approval and consent to participate: The study was approved by the ethics committee of the University of Campania "L. Vanvitelli". Clinical investigations were conducted according to the principles expressed in the Declaration of Helsinki.

Consent for participation and publication: All of patients gave their written consent for participation and publication.

Availability of data and materials: please contact authors for data request

Competing interests: The authors declare that they have no competing interests.

Funding: This research did not receive any specific grant from funding agencies in the public, commercial, or not-for-profit sectors

Paolo De Blasiis, Mariarosa A.B. Melone and Simone Sampaolo thank to the Inter-University Centre for Research in Neurosciences and University of Campania "Luigi Vanvitelli," project V:ALERE 2019 Id343-TRANSITION “Nutri-epigenetics and physical activity: a natural help for Neurofibromatosis type 1" Naples, Italy, for financial support.

Giacomo Lus received speaker honoraria and/or consultancy from Biogen, Teva, Genzyme, Merck, Novartis, Almirall, Roche.

Chiara Terracciano, Allegra Fullin, Mario Sansone have nothing to disclose.

Author contributions: Paolo De Blasiis and Simone Sampaolo conceived and designed the study. Paolo De Blasiis, Filomena Napolitano and Chiara Terracciano recruited the patients and performed clinical assessments. Paolo De Blasiis, Mario Sansone and Allegra Fullin contributed to the acquisition, analysis and interpretation of the data. Paolo De Blasiis and Simone Sampaolo drafted the first article. Simone Sampaolo, Maria Rosa Anna Beatrice Melone, Giacomo Lus and Luca Del Viscovo have critically revised the content. All authors have read and approved final version of the manuscript.

Acknowledgements: not applicable

\section{References}

1. Sampaolo S, Di lorio G., et al. Distinct disease phenotypes linked to different combinations of GAA mutations in a large late-onset GSDII sibship. Orphanet journal of rare diseases; (2013) 8:159. DOI: 10.1186/1750-1172-8-159

2. Case L.E., Beckemeyer A., Kishnani P.S. Infantile Pompe Disease on ERT-Update on Clinical Presentation, Musculoskeletal Management, and Exercise Considerations. Am J Med Genet C Semin Med Genet. 2012 Feb 15;160C (1):69-79. DOI: 10.1002/ajmg.c.31321

3. E.J. Feeney, et al., The value of muscle biopsies in Pompe disease: identifying lipofuscin inclusions in juvenile- and adult-onset patients, Acta Neuropathol. Commun. 2 (2014) 2. DOI: 10.1186/2051-5960-2-2

4. D. Gungor, et al., Enzyme replacement therapy and fatigue in adults with Pompe disease, Mol. Genet. Metab. 109 (2) (2013) 174-178. DOI: 10.1016/j.ymgme.2013.03.016

5. Favejee MM, van den Berg LE, Kruijshaar ME, et al. Exercise training in adults with Pompe disease: the effects on pain, fatigue and functioning. Arch Phys Med Rehabil 2015; 96:817-22. DOI: 10.1016/j.apmr.2014.11.020

6. P.S. Kishnani, et al., Methods of diagnosis of patients with Pompe disease: Data from the Pompe Registry, Mol. Genet. Metab. 113 (1-2) (2014) 84-91. DOI: 10.1016/j.ymgme.2014.07.014 
7. Kishnani C.J. The emerging phenotype of late onset Pompe disease. A systematic literature review. Molecular, genetics and metabolism. (2017) 120(3):163-172. DOI: 10.1016/j.ymgme.2016.12.004

8. Schuller A. et al. Toward deconstructing the phenotype of Late-Onset Pompe Disease. American Journal of Medical Genetics Part C. 160C:80-88. (2012) 17 January. DOI:10.1002/ajmg.c.31322

9. Figueroa-Bonaparte et al. Muscle MRI findings in childhood/adult onset Pompe disease correlate with muscle function. (2016) Plos one. DOI: 10.1371/journal.pone.0163493

10. Roberts M. et al. The prevalence and impact of scoliosis in Pompe disease: lessons learned from the Pompe Registry. Molecular genetics and metabolism. (2011) 104(4):574-82. DOI: 10.1016/j.ymgme.2011.08.011

11. Valle M.S. et al. Quantitative analysis of upright standing in adults with Lopd. Scientific Reports. (2016) Volume 6, Article number: 37040. doi: $10.1038 /$ srep37040

12. Kadaba M. P., Ramakrishnan H. K., Wootten M. E. “Measurement of lower extremity kinematics during level walking”. Journal of Orthopaedic Research, Vol. 8, No. 3, pp. 383-39 (1990). DOI: 10.1002/jor.1100080310

13. R Core Team (2018). R: A language and environment for statistical computing. R Foundation for Statistical Computing, Vienna, Austria. URL https://www.R-project.org/.

14. Van der Ploeg A. et al. Prospective exploratory muscle biopsy, imaging, and functional assessment in patients with late-onset Pompe disease treated with al glucosidase alfa: The EMBASSY Study. Molecular Genetics and Metabolism (2016) 119. 115-123. DOI: 10.1016/j.ymgme.2016.05.013

15. Wokke J.H.J. et al. Clinical features of late-onset Pompe disease: a prospective cohort study. Muscle Nerve (2008) 38: 1236-1245. DOI: $10.1002 /$ mus. 21025

16. McGregor M. The significance of certain measurements of the skull in the diagnosis of basilar impression. The British journal of radiology. (1948) 21:171-181. DOI: 10.1259/0007-1285-21-244-171

17. Berthonnaud E. et al. Analysis of the Sagittal Balance of the Spine and Pelvis Using Shape and Orientation Parameters. Journal of Spinal Disorders \& Techniques. (2005) Volume 18 - Issue 1 - p 40-47. DOI: 10.1097/01.bsd.0000117542.88865.77

18. Kuntz C. IV, Levin L. S., Ondra S. L., Shaffrey C. I. and Morgan C. J. Neutral upright sagittal spinal alignment from the occiput to the pelvis in asymptomatic adults: a review and resynthesis of the literature. Journal of neurosurgery Spine. (2007) Feb;6(2):104-12. DOI:10.3171/spi.2007.6.2.104

19. Zheng zeng, Yong Hai, Yunfeng Bi, Bing Wang, Miaomiao liu and Yang Liu. Characteristics of sagittal spinopelvic alignment in asymptomatic Han Chinese adults. Experimental and therapeutic medicine (2018) 16: 4107-4113. DOI: 10.3892/etm.2018.6680

20. Hasegawa K., Okamoto M., Hatsushikano S., Shimoda H., Ono M., Watanabe K. Normative values of spino-pelvic sagittal alignment, balance, age, and health-related quality of life in a cohort of healthy adult subjects. European spine journal. (2016) Nov;25(11):3675-3686. DOI: $10.1007 /$ s00586-016-4702-2

21. Roussouly P., MD, Sohrab Gollogly, MD, Eric Berthonnaud, PhD, and Dimnet J., PhD. Classification of the Normal Variation in the Sagittal Alignment of the Human Lumbar Spine and Pelvis in the Standing Position. Spine (Phila Pa 1976). (2005) 30(3):346-53. DOI: 10.1097/01.brs.0000152379.54463.65

22. Roussouly P., Nnadi C. Sagittal plane deformity: an overview of interpretation and management. European Spine Journal. (2010) Volume 19, pages1824-1836. DOI: 10.1007/s00586-010-1476-9

23. Gelb D.E., Lenke L.G., Bridwell K.H., Blanke K., McEnery K.W. An analysis of sagittal spinal alignment in 100 asymptomatic middle and older aged volunteers. (1995) Spine Volume 20.

24. Kine E., D'Amico M., Roncoletta P. Normative 3D opto-electronic stereophotogrammetric sagittal alignment parameters in a young healthy adult population. Plose One, (2018) 13(9). //doi.org/10.1371/journal.pone.0203679

25. Benedetti M.G., Catani F., Leardini A., Pignotti E., Giannini S. Data management in gait analysis for clinical applications. (Bristol, Avon). Clinical Biomechanics 13. (1998) Apr;13 (3):204-215. DOI: 10.1016/s0268-0033(97)00041-7

26. Wun g et al. ISB recommendation on definitions of joint coordinate system of various joints for the reporting of human joint motion-part I: ankle, hip, and spine. Journal of Biomechanics. Vol 35, Issue 4, April (2002), Pages 543-548. doi: 10.1016/s0021-9290(01)00222-6.

\section{Tables}

Table.1 MRC and ROM values. Median, mean, moda and Standard Deviation were reported for each parameter of patients and controls. Significant differences ( $\mathrm{p}$-value<0.05) between patients and controls for ROM values were highlighted 


\begin{tabular}{|c|c|c|c|c|c|c|c|c|c|c|c|c|c|c|c|c|}
\hline & $\begin{array}{l}\text { Trunk } \\
\text { ext }\end{array}$ & $\begin{array}{l}\text { Trunk } \\
\text { flex }\end{array}$ & $\begin{array}{l}\text { Hip } \\
\text { ext. }\end{array}$ & $\begin{array}{l}\text { Hip } \\
\text { flex }\end{array}$ & $\begin{array}{l}\text { Hip } \\
\text { abd. }\end{array}$ & $\begin{array}{l}\text { Hip } \\
\text { add. }\end{array}$ & $\begin{array}{l}\text { Knee. } \\
\text { ext }\end{array}$ & $\begin{array}{l}\text { Knee } \\
\text { flex. }\end{array}$ & $\begin{array}{l}\text { DF } \\
\text { TA }\end{array}$ & ELA & ECD & $\mathrm{PF}$ & FLA & FLD & $\begin{array}{l}\text { a- } \\
\text { ROM } \\
\text { K ex }\end{array}$ & $\begin{array}{l}\text { p- } \\
\text { ROM } \\
\text { K ex }\end{array}$ \\
\hline 1 & 3 & 4 & 4 & 4 & 4 & 4 & 5 & 4 & 2 & 3 & 4 & 5 & 4 & 5 & -10 & 0 \\
\hline 2 & 2 & 2 & 4 & 4 & 4 & 4 & 5 & 4 & 3 & 4 & 5 & 5 & 3 & 5 & 5 & 10 \\
\hline 3 & 2 & 2 & 5 & 3 & 3 & 3 & 4 & 3 & 1 & 4 & 5 & 5 & 4 & 4 & -25 & -10 \\
\hline 4 & 4 & 4 & 4 & 4 & 4 & 4 & 5 & 4 & 3 & 4 & 4 & 5 & 4 & 4 & 10 & 15 \\
\hline 5 & 2 & 2 & 5 & 4 & 4 & 4 & 4 & 4 & 2 & 2 & 4 & 5 & 3 & 4 & -25 & -5 \\
\hline 6 & 2 & 3 & 5 & 3 & 3 & 4 & 5 & 3 & 2 & 4 & 5 & 5 & 4 & 5 & -5 & 15 \\
\hline 7 & 2 & 2 & 4 & 4 & 4 & 4 & 4 & 3 & 4 & 4 & 5 & 5 & 4 & 4 & 10 & 20 \\
\hline (median) & 2 & 2 & 4 & 4 & 4 & 4 & 5 & 4 & 2 & 4 & 5 & 5 & 4 & 4 & -5 & 10 \\
\hline (mean) & N.A. & N.A. & N.A. & N.A. & N.A. & N.A. & N.A. & N.A. & N.A. & N.A. & N.A. & N.A. & N.A. & N.A. & -5.7 & 6.4 \\
\hline (moda) & 2 & 2 & 4 & 4 & 4 & 4 & 5 & 4 & 2 & 4 & 5 & 5 & 4 & 4 & -25 & 15 \\
\hline (SD) & N.A. & N.A. & N.A. & N.A. & N.A & N.A. & N.A. & N.A. & N.A. & N.A. & N.A. & N.A. & N.A. & N.A. & & 11.4 \\
\hline Controls & 5 & 5 & 5 & 5 & 5 & 5 & 5 & 5 & 5 & 5 & 5 & 5 & 5 & 5 & 20 & 25 \\
\hline P-value & N.A. & N.A. & N.A. & N.A. & N.A. & N.A. & N.A. & N.A. & N.A. & N.A. & N.A. & N.A. & N.A. & N.A. & 0.002 & 0.003 \\
\hline
\end{tabular}

Table note: flexion (flex), extension (ext); dorsiflexion (DF), Tibialis Anterior (TA); extensor longus hallucis (ELA), Extensor communis digitorum (ECD), muscle ankle Plantiflexor (PF), Flexor Longus hallucis (FLA), Flexor Longus Digitorum (FLD), Active (a) and passive (p)- Range of Movement (ROM) with extended knee

Table.2 Mean and standard deviation of stereo-photogrammetric parameters in Helen Hayes M.M. and DB Total Protocol were reported for each parameter and the significant differences ( $p$-value<0.05) between the two groups are highlighted. 


\begin{tabular}{|c|c|c|c|}
\hline & $\begin{array}{l}\text { LOPD } \\
(\text { mean } \pm \mathrm{SD})\end{array}$ & $\begin{array}{l}\text { Healthy Controls } \\
\text { (mean } \pm \text { SD) }\end{array}$ & $\mathrm{p}$-value \\
\hline \multicolumn{4}{|c|}{ Parameters in Helen Hayes standard protocol } \\
\hline St-TT $\left[^{\circ}\right]$ & $31.3 \pm 10.3$ & $34.0 \pm 9.1$ & 0.670 \\
\hline St-PT $\left[^{\circ}\right]$ & $7.8 \pm 5.9$ & $11.9 \pm 3.7$ & 0.061 \\
\hline St-HFE Right $\left[{ }^{\circ}\right]$ & $5.6 \pm 8.3$ & $5.4 \pm 5.2$ & 0.480 \\
\hline St-HFE Left $\left[^{\circ}\right]$ & $5.6 \pm 6.5$ & $4.8 \pm 4.3$ & 0.381 \\
\hline St-KFE Right $\left[{ }^{\circ}\right]$ & $6.7 \pm 9.7$ & $-0.4 \pm 5.6$ & 0.071 \\
\hline St-KFE Left [ [] & $7.1 \pm 9.2$ & $-1.7 \pm .6$ & 0.018 \\
\hline St-AFE Right $\left[{ }^{\circ}\right]$ & $7.5 \pm 2.1$ & $3.9 \pm 3.3$ & 0.039 \\
\hline St-AFE Left $\left[^{\circ}\right]$ & $6.9 \pm 2.4$ & $3.0 \pm 3.5$ & 0.039 \\
\hline \multicolumn{4}{|c|}{ Additional parameters in DB Total protocol } \\
\hline St-CNA $\left[^{\circ}\right]$ & $49.9 \pm 5.2$ & $53.2 \pm 7.2$ & 0.072 \\
\hline St-SCA $\left[^{\circ}\right]$ & $0.1 \pm 3.9$ & $3.9 \pm 1.9$ & 0.015 \\
\hline St-STA [] & $-3.1 \pm 7.1$ & $-5.2 \pm 3.2$ & 0.257 \\
\hline St-SLA [] & $12.5 \pm 7.9$ & $16.1 \pm 7.2$ & 0.196 \\
\hline St-HSA $\left[^{\circ}\right]$ & $0.9 \pm 2.5$ & $1.2 \pm 1.4$ & 0.270 \\
\hline St-CT $\left[^{\circ}\right]$ & $47.2 \pm 16.3$ & $54.2 \pm 12.9$ & 0.330 \\
\hline St-DA [ ["] & $164.5 \pm 11.2$ & $151.4 \pm 5.5$ & 0.005 \\
\hline St-LA [] & $188.8 \pm 14.5$ & $198.6 \pm 8.7$ & 0.231 \\
\hline St-SVA [m] & $0.00 \pm 0.03$ & $0.03 \pm 0.02$ & 0.004 \\
\hline St-EF right $\left[^{\circ}\right]$ & $156.5 \pm 9.3$ & $155.9 \pm 5.5$ & 0.100 \\
\hline St-EF Left [º] & $160.2 \pm 2.5$ & $156.5 \pm 6.5$ & 0.014 \\
\hline St-WSO [m] & $-0.1 \pm 0.04$ & $-0.2 \pm 0.3$ & 0.090 \\
\hline St-HSN $\left[^{\circ}\right]$ & $164.8 \pm 3.9$ & $161.2 \pm 2.8$ & 0.031 \\
\hline St-HSC [ [] & $180.8 \pm 4.7$ & $177.3 \pm 2.4$ & 0.023 \\
\hline St-HST $\left[^{\circ}\right]$ & $183.9 \pm 8.8$ & $186.4 \pm 3.3$ & 0.560 \\
\hline St-SPA $\left[^{\circ}\right]$ & $131.8 \pm 10.6$ & $133.3 \pm 6.2$ & 0.357 \\
\hline
\end{tabular}

Table. 3 Mean and standard deviation of radiographic values were reported, the significant differences between the two groups were highlighted ( $p$-value<0.05). Normal values were reported in article $[16,17,18,19]$. 


\begin{tabular}{|c|c|c|c|c|}
\hline & $\begin{array}{l}\text { LOPD } \\
(\text { mean } \pm S D)\end{array}$ & $\begin{array}{l}\text { Normal values } \\
\text { (mean } \pm \text { SD) }\end{array}$ & & p-value \\
\hline $\mathrm{O}-\mathrm{C} 2\left[^{\circ}\right]$ & $7.22 \pm 1.79$ & $14.00 \pm 7.14$ & [16] & $<0.001$ \\
\hline $\mathrm{C} 2-\mathrm{C} 7 \mathrm{CA}\left[^{\circ}\right]$ & $13.62 \pm 14.78$ & $0.2 \pm 2$ & [17] & 0.031 \\
\hline C-DA $\left[^{\circ}\right]$ & $25.5 \pm 19.4$ & $47.5 \pm 4.8$ & [17] & 0.019 \\
\hline C-LA $\left[^{\circ}\right]$ & $33.2 \pm 18.6$ & $42.7 \pm 5.4$ & [17] & 0.134 \\
\hline cvb-DA $\left[^{\circ}\right]$ & $160.6 \pm 9.2$ & NA & & NA \\
\hline cvb-LA [ [0] & $192.3 \pm 10.1$ & NA & & NA \\
\hline Mk-DA $\left[^{\circ}\right]$ & $162.4 \pm 8.59$ & NA & & NA \\
\hline Mk-LA [0] & $161.1 \pm 8.46$ & NA & & NA \\
\hline L5-S1 [º] & $26.00 \pm 13.82$ & $24.00 \pm 6.12$ & [18] & 0.642 \\
\hline L1-S1 [] & $61.40 \pm 13.09$ & $62.00 \pm 11.22$ & [18] & 0.454 \\
\hline SVA [m] & $-0.02 \pm 0.03$ & $0.00 \pm 0.02$ & [18] & 0.082 \\
\hline SPA $\left[^{\circ}\right]$ & $164.8 \pm 11.3$ & NA & & NA \\
\hline SSA $\left[^{\circ}\right]$ & $127.8 \pm 16.3$ & $122.9 \pm 7.8$ & [19] & 0.751 \\
\hline SS $\left[^{\circ}\right]$ & $34.2 \pm 14.9$ & $39.7 \pm 4.1$ & [17] & 0.204 \\
\hline $\mathrm{PT}\left[\left[^{\circ}\right]\right.$ & $17.6 \pm 13.9$ & $12.1 \pm 3.2$ & [17] & 0.812 \\
\hline $\mathrm{PI}\left[^{\circ}\right]$ & $51.9 \pm 18.2$ & $51.8 \pm 5.3$ & [17] & 0.503 \\
\hline
\end{tabular}

Table note: Markers (Mk), Centre of Vertebral Body (cvb), kyphosis (K), Lordosis (L), Dorsal Angle (DA), Lumbar Angle (LA); Non-available (NA).

Table.4 Correlation coefficients with Confidence Intervals $(\mathrm{Cl})$ between radiographic and Stereo-photogrammetric parameters: Mk- and cvbangles, Mk- and St- angles, cvb- and St- angles; Mk and Cobb angles, St and Cobb angles; St-SVA and SVA. The strong and significant correlations between parameters the two groups are highlighted (correlation coefficient $>0.7$ and $<-0.7 ; p$-value $<0.05$ ).

\begin{tabular}{|lll|}
\hline Sagittal parameters & $\begin{array}{l}\text { Correlation coefficients } \\
\text { (Confidence interval }(\mathrm{Cl}))\end{array}$ & p-value \\
\hline Mk-DA vs St-DA & $\mathbf{0 . 9 7}(\mathbf{0 . 7 3} ; \mathbf{0 . 9 9 )}$ & $\mathbf{0 . 0 0 1 5}$ \\
\hline Mk-LA vs St-LA & $\mathbf{0 . 8 1}(\mathbf{0 . 0 2} ; \mathbf{0 . 9 8 )}$ & $\mathbf{0 . 0 4 7 0}$ \\
\hline St-DA vs cvb-DA & $\mathbf{0 . 9 2}(\mathbf{0 . 4 3} \mathbf{0 . 9 9 )}$ & $\mathbf{0 . 0 0 9 3}$ \\
\hline St-LA vs cvb-LA & $0.29(-0.68 ; 0.89)$ & 0.5834 \\
\hline Mk-DA vs cvb-DA & $\mathbf{0 . 9 9 ( 0 . 8 8 ; 0 . 9 9 )}$ & $<\mathbf{0 . 0 0 1}$ \\
\hline Mk-LA vs cvb-LA & $0.67(-0.32 ; 0.96)$ & 0.1493 \\
\hline St-SVA vs SVA & $0.50(-0.52 ; 0.93)$ & 0.3102 \\
\hline Mk-DA vs C-DA & $-0.80(0.055 ;-0.98)$ & $\mathbf{0 . 0 0 3}$ \\
\hline Mk-LA vs C-LA & $-0.74(0.090 ;-0.90)$ & 0.170 \\
\hline St-DA vs C-DA & $-0.71(-0.965 ; 0.238)$ & 0.113 \\
\hline St-LA vs C-LA & $-0.49(-0.93 ; 0.54)$ & 0.325 \\
\hline
\end{tabular}

Table note: Stereophotogrammetric (St), Markers (Mk), Centre of Vertebral Body (cvb), Cobb Angle dorsal (C-DA), Cobb Lumbar Angle (C-LA), Dorsal Angle (DA), Lumbar Angle (LA).

\section{Figures}



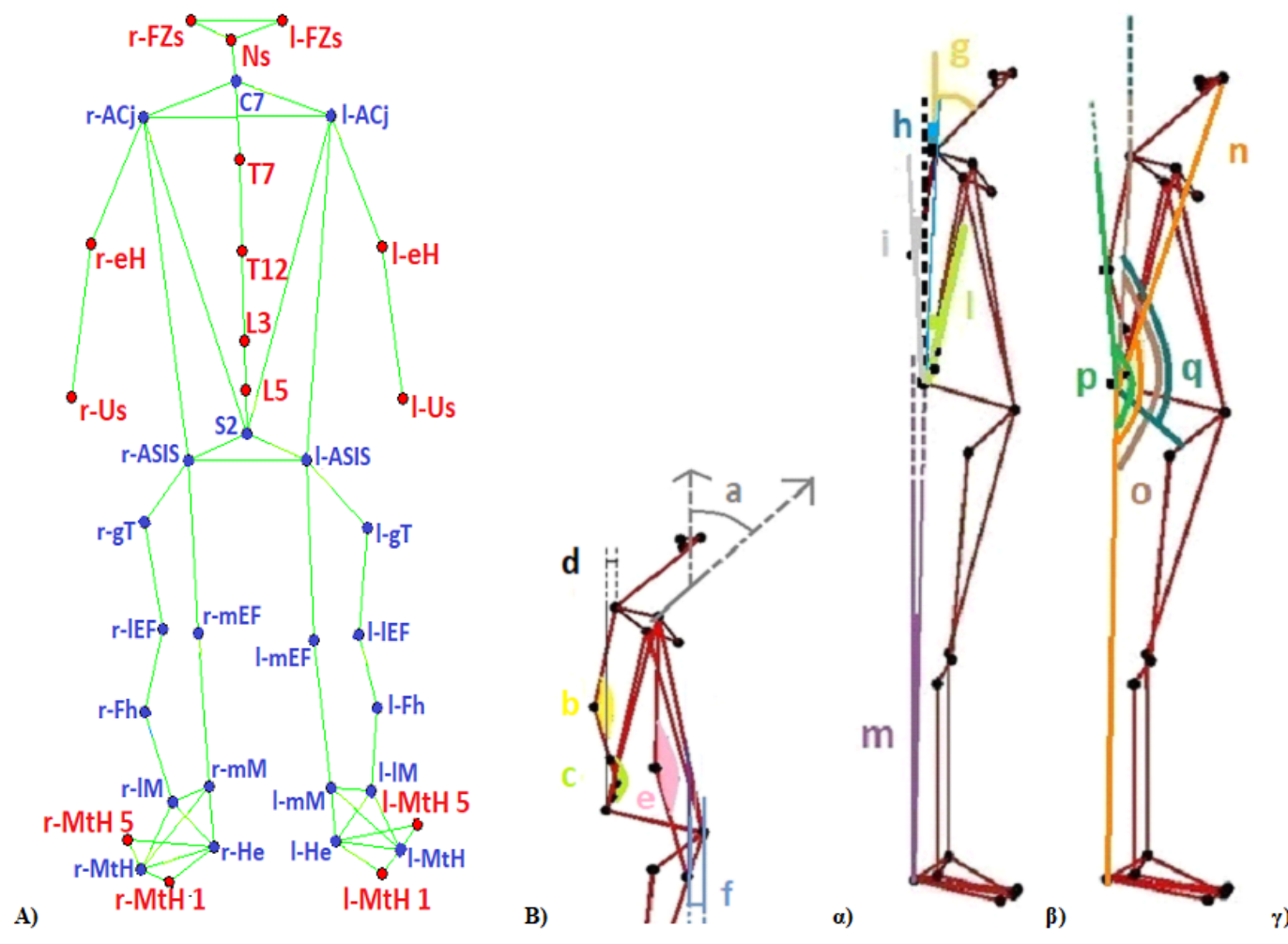

\begin{tabular}{|c|c|c|c|c|}
\hline \multicolumn{5}{|c|}{ Param eters in Image B- $\alpha$} \\
\hline a & St-CT & Cervical Tilt $\left[{ }^{\circ}\right]$ & The angle between the: & cervical plane (r-FZs, Ns, 1-FZs) and trunk plane (C7, r-ACj, 1-ACj). \\
\hline $\mathrm{b}$ & St-DA & Dorsal Angle [०] & \multirow{2}{*}{$\begin{array}{l}\text { The anterior angle } \\
\text { between the: }\end{array}$} & strai ght line joining $\mathrm{C} 7$ to $\mathrm{T} 7$ and the str aight line joining $\mathrm{T} 7$ to $\mathrm{T} 12$. \\
\hline $\mathrm{c}$ & St-LA & Lumbar Angle [ $\left.{ }^{\circ}\right]$ & & strai ght line joining $T 12$ to $L 3$ and the straight line joining L 3 to L5. \\
\hline d & St-SVA & Sagittal Vertical Axis [m] & The distance from: & S1- to a plumb line dropped from $\mathrm{C} 7$. \\
\hline $\mathrm{e}$ & St-EF & Elbow Flexion $\left[{ }^{\circ}\right]$ & The angle between the: & straight line joining Shoulder to Elbow and the straight line joining the Elbow to Wrist \\
\hline f & St-WSO & Wrist-ASIS Offset [m] & The distance from: & wris to a plumb line dropped from Anterior Superior Iliac Spine. \\
\hline \multicolumn{5}{|c|}{ Parameters in Image B- $\beta$} \\
\hline $\mathrm{g}$ & St-CNA & C7-Nasion Angle [ ${ }^{\circ}$ ] & \multirow[t]{5}{*}{ The angle between the: } & straight line joining $\mathrm{C} 7$ to Nasion and the vertical axis (from $\mathrm{C} 7$ ). \\
\hline $\mathrm{h}$ & St-SCA & S2-C7 Angle [ $\left.{ }^{\circ}\right]$ & & strai ght line joiring $\mathrm{S} 2$ to $\mathrm{C} 7$ and the vertical ax is (from $\mathrm{S} 2$ ). \\
\hline $\mathrm{i}$ & St-STA & S2-T7 Angle $\left[{ }^{\circ}\right]$ & & strai ght line joining $\mathrm{S} 2$ to $\mathrm{T} 7$ and the vertical axis (from $\mathrm{S} 2$ ). \\
\hline 1 & St-SLA & S2-L5 Angle [ $\left.{ }^{\circ}\right]$ & & strai ght line joining S2 to L5 and the vertical axis (from S2). \\
\hline & St-HSA & Heel-S2 Angle $\left[{ }^{\circ}\right]$ & & straight line joining Heel to $\mathrm{S} 2$ and the vertical ax is (fr om Heel). \\
\hline \multicolumn{5}{|c|}{ Param eters in Image B- $\gamma$} \\
\hline $\mathrm{n}$ & St-HSN & Heel-S2-Nasion Angle ["] & \multirow{4}{*}{$\begin{array}{l}\text { The anterior angle } \\
\text { between the: }\end{array}$} & straight line joiring Heel to $\mathrm{S} 2$ and the straight line joining the $\mathrm{S} 2$ to Nasion. \\
\hline 0 & St-HSC & Heel-S2-C7 Angle [ $\left.{ }^{\circ}\right]$ & & straight line joining Heel to $\mathrm{S} 2$ and the straight line joining the $\mathrm{S} 2$ to $\mathrm{C} 7$. \\
\hline $\mathrm{p}$ & St-HST & Heel-S2-T7 Angle [ $\left.{ }^{\circ}\right]$ & & straight line joining Heel to $\mathrm{S} 2$ and the straight line joining the $\mathrm{S} 2$ to $\mathrm{T} 7$. \\
\hline $\mathrm{q}$ & St-SPA & Spino Pelvic Angle $\left[{ }^{\circ}\right]$ & & line $\mathrm{C} 7$-S2 and $\mathrm{S} 2$-Center of Hip Axis. \\
\hline
\end{tabular}

\section{Figure 1}

A) DB Total markers placement protocol shown on stick diagram indicating the bodily segments elaborated by tracking of software BTS (label of landmarks); B) DB Total stick diagrams associated to calculated sagittal parameters; C) Definitions of stereophotogrammetric parameters. Figure note: Stereofotogrammetric (St). Standard HHMM protocol [12] markers in blue and DB Total markers in red: nasion (Ns), frontozygomatic suture (FZs), spiny apophysis of C7 -T7 -T12 -L3 -L5 -S2, acromioclavicular joint (ACj), epicondylus humeri (eH), ulnar styloid (Us), anterior-superior iliac spine (ASIS), greater trochanter ( $\mathrm{gT}$ ), medial (mEF) and lateral (IEF) epicondylitis femoris, fibular head (Fh) , medial $(\mathrm{mM})$ and lateral $(\mathrm{IM})$ malleolus, $\mathrm{I}^{\circ}-\mathrm{III}^{\circ}$ and $\mathrm{V}^{\circ}$ metatarsal head $(\mathrm{MtH})$, heel $(\mathrm{He})$. 


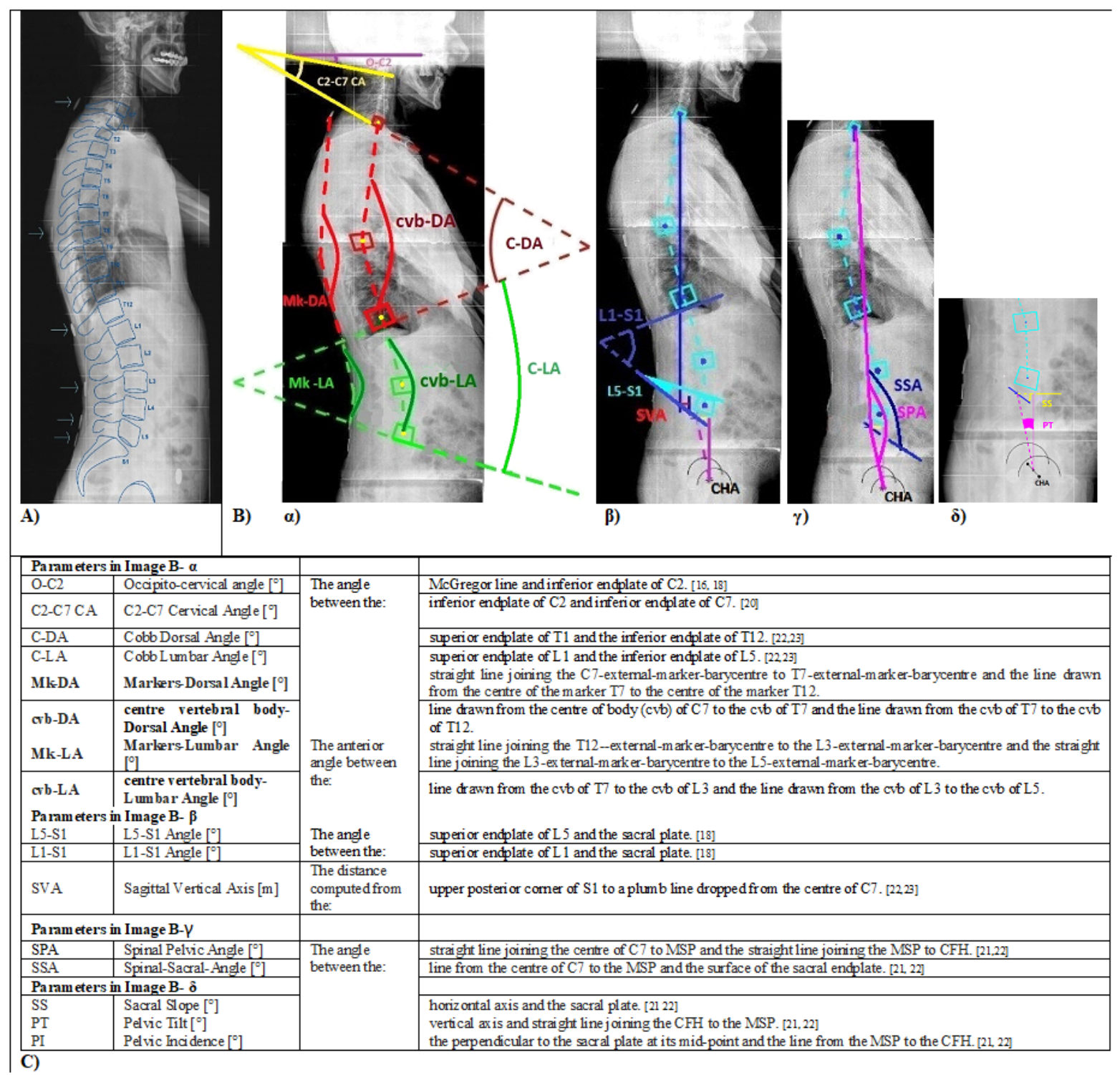

\section{Figure 2}

A) x-Ray of sagittal spine, highlighting vertebral body, spiny apophysis and radiopaque markers placed on C7-T7-T12-L3-L5-S2; B) Radiographic parameters calculated with Matlab script; C) Definitions of radiological parameters. Figure note: (x-Ray of two different patients) MSP: mid-point of the sacral plate; $\mathrm{CFH}$ : centre of the femoral heads.

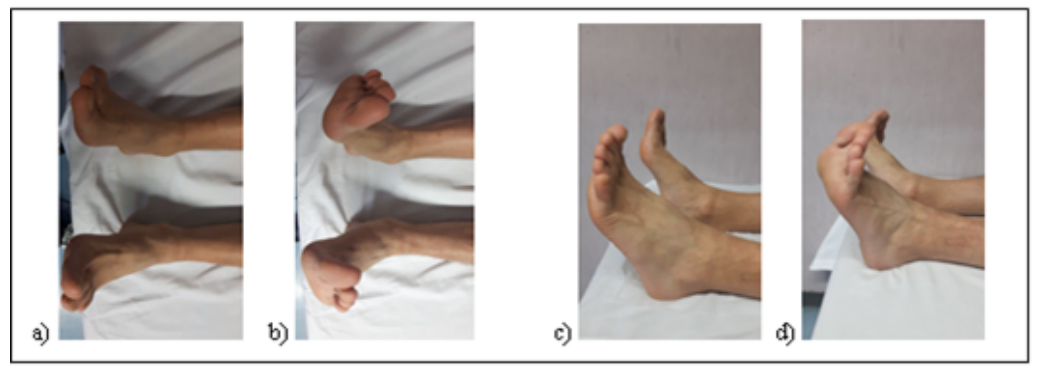

Figure 3

Typical morphology of LOPD patients' feet at rest $(a, c)$ and during active dorsiflexion $(b, d)$ on frontal $(a, b)$ and sagittal $(c, d)$ plane. 